\title{
Thermocapillary micromanipulation: force characterization and Cheerios interactions
}

\author{
Ronald Terrazas • Adrien De Maeijer . \\ Aude Bolopion • Michaël Gauthier . \\ Michel Kinnaert • Pierre Lambert
}

Received: date / Accepted: date

\begin{abstract}
Thermocapillary micromanipulation is an emerging non-contact micromanipulation technique, allowing to displace particles in the liquid bulk or at the free surface. When the particles are at the free surface and the surface is heated from the top, the actuation force is repulsive and not attractive. The handling technique is then intrinsically unstable. Therefore, control schemes have been reported recently to deal with this instability. They are based on an experimental characterization of the physical system (depending on the laser, the liquid and the particle properties). In this paper, we explain how we could make use of these handling schemes to estimate the thermocapillary force developed by the laser on the particle to be about $8 \mathrm{nN}$. This work is a first step towards the handling of multiple particles at the air/liquid interface.
\end{abstract}

Keywords Thermocapillary micromanipulation · capillary forces · optofluidics · feedforward · capillary dipole · Cheerios effect

\section{Introduction}

Non-contact micromanipulation, which consists in controlling objects based on remotely induced force fields, has received increasing interest these last years. The major advantages of this approach are the fact that it can be used in confined environments where direct access is complex and that the lack

Ronald Terrazas, Adrien De Maeijer and Pierre Lambert

TIPs Dpt, Université libre de Bruxelles, 50 av. Roosevelt, B-1050 Brussels

E-mail: pierre.lambert@ulb.ac.be

Aude Bolopion and Michaël Gauthier

AS2M dpt, FEMTO-ST, 24 Rue Savary, F-25000 Besançon

E-mail: aude.bolopion@femto-st.fr, michael.gauthier@femto-st.fr

Michel Kinnaert

SAAS Dpt, Université libre de Bruxelles, 50 av. Roosevelt, B-1050 Brussels

E-mail: michel.kinnaert@ulb.ac.be 
of direct contact with the object reduces greatly the risk of contamination. Different physical principles have been investigated to generate the force fields, such as magnetophoresis [1], dielectrophoresis[2], acoustophoresis [3] or optical effects [4].

Another very promising actuation principle has been proposed, based on the thermocapillary effect. It consists in heating a fluid locally to generate a temperature gradient along its interface (with air or with another fluid). This induces a surface stress since surface tension depends on temperature (Marangoni effect). This stress is then used to trigger flows to displace particles. This manipulation technique has been compared in [5] to other classical non-contact actuation technique. This study concludes that the thermocapillary effect enables to reach large velocities and can be a promising alternative to classical approaches.

The thermocapillary effect can produce either an attractive force field or a repulsive one since it induces convection cells [6]. Thus, several micromanipulation approaches have been reported using this principle. In [7], a single gold nanoparticle is transported by heating the surface of the fluid from the top. The temperature increase is less than $2^{\circ}$, but velocities in the order of $\mu \mathrm{m} / \mathrm{s}$ are reached. In $[8,9], 1-100 \mu \mathrm{m}$ particles are moved in the bottom of a water reservoir by heating the water from the bottom with a laser. In [10], the generation of bubbles in oil is controlled, which in turn creates flows in the oil, able to move particles. A similar approach was applied to bubbles in water [11].

In [12] it is proposed to manipulate micrometric particles floating at the interface between water and air, using a laser heating from the top. It emphasizes that such a system configuration can generate fast and localized flows with limited temperature increase (few degrees only) which allows to exert significant forces on the particles. Particles manipulated at the interface (i.e. the free water-air surface) move faster than particles manipulated in the fluid bulk [13]. Particle velocities up to several $\mathrm{mm} / \mathrm{s}$ are reported. From the applicative point of view, the manipulation at the interface is of interest: the assembly or conveying of fragile objects that require planar displacements only will greatly benefit from non-contact actuation techniques at the interface. Controlled manipulation can also provide a powerful tool to better understand the physics at the interface, or the interactions between particles. Several works report such interest [14-18]. However, the manipulation at the air/water interface presents several drawbacks: it requires proper wetting conditions for the particles to float and remain at the fluid interface, and the particles are repelled from the laser, which makes the system unstable. A closed-loop controller allows to control this unstable system and also improves the performance of the system [19].

Many applications require to manipulate several particles simultaneously and independently, for example to perform assembly of components, or to manipulate objects indirectly by using several microrobots that make a cage around them [20]. However, multiple particles handling remains challenging. It has been addressed in the case of opto-thermocapillary flow-addressed bubble 
microrobots, where bubbles at the bottom of a reservoir are manipulated using laser heating from the bottom of the reservoir [21]. Up to 50 microrobots are manipulated in parallel using holographic techniques [22]. However, multiple particles handling at the air-water interface presents different issues, such as the capillary interactions between them (known as the Cheerios effect). We published a strategy to reject the capillary perturbation induced by a neighboring particle thanks to a feedforward control scheme [23]. This article is a follow-up of the MARSS conference article [23]. Therefore, parts of this article have been directly reproduced from [23] with permission. The new contribution of this manuscript is the use of these developed tools to estimate experimentally the thermocapillary force developed on the particle by the thermal gradient induced by the absorption of a laser beam. This work is thus the first step toward simultaneous control of several microrobots at the interface.

This paper is organized as follows. A brief introduction to thermocapillary micromanipulation is presented in Section 2. The physical principles are recalled, and the experimental setup is presented. The capillary forces are presented in Section 3. Section 4 presents an original feedforward controller dedicated to the manipulation of particles in the presence of obstacles, based on the feedback controller presented in [23]. Section 5 gives the results obtained both in simulation and experimentally. Finally Section 6 concludes the paper and discusses future works.

\section{Thermocapillary micromanipulation}

\subsection{Principle of thermocapillary micromanipulation}

The physical principle of thermocapillary manipulation has been extensively detailed in [13]. Only the basic principles are given here. The particles to be displaced are put on the surface of a water layer. Initially the water is at rest at ambient temperature. At time $t=0$, an IR laser coming from atop is directed toward the water and therefore absorbed by the liquid according to Beer-Lambert's law.

The absorbed energy is converted into heat, which propagates in the liquid (first by diffusion). This creates a temperature gradient at the interface which generates a surface tension stress at the interface. This triggers fluid motion and additional heat propagation by convection. From there, the velocity field across the entire fluid can be computed. The particle placed at the interface will follow the fluid movement due to the drag force, and will thus be displaced.

\subsection{Experimental setup}

The general scheme of the experimental setup dedicated to thermocapillary micromanipulation is shown in Fig.1. The setup consists of an IR laser system able to control the location of the laser using a mirror and a camera to track 
the object position. The entire setup is mounted on an anti-vibration table. To heat the water a continuous-wave (CW) Keopsys Fiber Raman Laser CRFL01-1455-OM1-B130-FA with a wavelength of $1455 \mathrm{~nm}$ is used. The laser power is set at $37 \mathrm{~mW}$. The beam diameter at the water surface is $1.2 \mathrm{~mm}$. At this wavelength, optical power is absorbed by water with an absorption coefficient $\alpha$ of $3275 \mathrm{~m}^{-1}$ [24]. Using Beer-Lambert law this means that $95 \%$ of the laser power is absorbed within a water layer of $900 \mu \mathrm{m}$, which is then transformed into heat. The temperature increase is between 1 and $2{ }^{\circ} \mathrm{C}$ at the location of the laser spot. However, the general averaged temperature increase in the whole water container is negligible. The water is ultra pure water, produced by a Sartorius machine, which meets ASTM Type 1 water quality standards.

Because the infrared laser is invisible to the human eye, it is coupled with a visible red laser using a laser coupler. The coupled beam is collimated and directed towards a plastic container filled with ultra pure water, using piezoactuated tip/tilt mirror whose tilt angle can be controlled. The mirror Mirrorcle Technologies A7M20.1 was used. This mirror has a large tip/tilt range of $\pm 87 \mathrm{mrad}$ on each axis (equivalent to $\pm 174 \mathrm{mrad}$ optical beam deflection). With this, a working space defined by a rhombus with diagonals 180 and 118 mm was obtained.

The camera (IDS camera UI-3370CP) which tracks the object position uses a lens with a given focal length in combination with an extensor ring in order to have a field of view equal or larger than the working space defined by the piezo tip/tilt mirror. In order to facilitate the image recognition, the container is placed on top of a white led backlight. This plastic container is covered using a plastic lid in order to reduce the surface contamination through time.

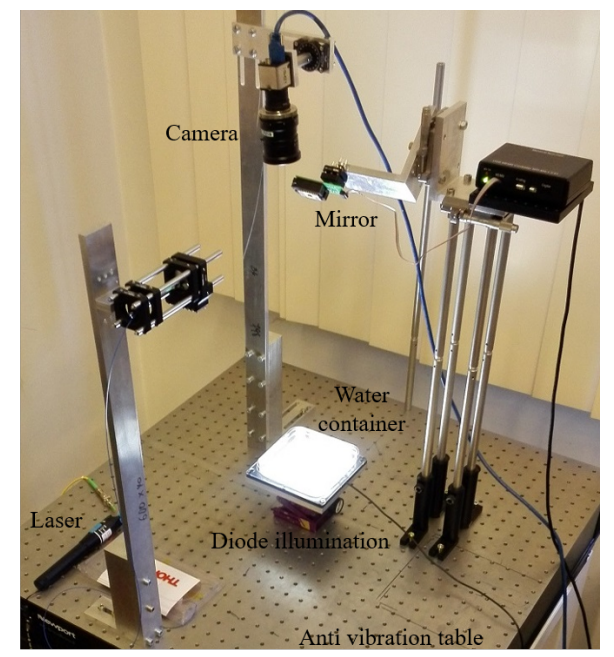

Fig. 1 Experimental setup. 
The objects that are manipulated are spherical AISI 304 steel spheres with $500 \mu \mathrm{m}$ diameter. They are placed at the air water interface. The balance of forces that ensures equilibrium for a particle staying on the surface of a liquid is $F_{\text {gravity }}+F_{h y d r}=F_{\sigma z}+F_{\text {buoyancy }}$ where $F_{\text {gravity }}$ corresponds to the weight of the particle [25]. The vertical component of surface tension force $\left(F_{\sigma z}\right)$ is the main force responsible for holding the particle at the interface with some help of the buoyancy force $F_{\text {buoyancy }}$. These two forces must counteract the effect of the particle weight and the hydrostatic pressure force $F_{h y d r}$. To determine if a particle will remain at the surface or not, the main issue is to determine the particle/fluid contact angle $\theta_{c}$. If it is too small, the particle is hydrophilic and will sink. Otherwise, the particles can stay at the surface, depending on their weight. A detailed study is performed in [12] to determine which particles can be used depending on their material and size.

\section{Lateral Capillary Forces}

When particles are placed close to each other at the air/liquid interface, capillary interactions occur. As shown in Fig. 2, lateral capillary forces acting on floating particles can be attractive or repulsive, according to the sign of the capillary dipoles $Q_{i}=r_{i} \sin \alpha_{i}$, where $r_{i}$ is the radius of the contact line (ie the separation line between the wet and dry zones of the floating sphere) and $\alpha_{i}$ is the slope of the liquid-air interface tangent. The sign can be determined by looking at Figure $2(\mathrm{~b}): \alpha_{1}$ is negative and $\alpha_{2}$ is positive [26].

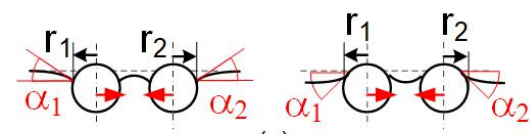

(a)

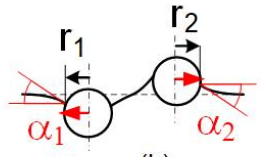

(b)

Fig. 2 Lateral capillary forces between particles [26]. a) Attracted particles with same wetting properties $\left(r_{1} \sin \alpha_{1} \cdot r_{2} \sin \alpha_{2}>0\right)$ (b) Repelled particles with opposite wetting properties $\left(r_{1} \sin \alpha_{1} \cdot r_{2} \sin \alpha_{2}<0\right)$.

Kralchevsky [26] proposed a model for the lateral capillary force $F_{\text {LatCap }}$ :

$$
F_{\text {LatCap }}=2 \pi \sigma Q_{1} Q_{2} L_{\text {cap }}^{-1} K_{1}\left(L_{\text {cap }}^{-1}\left\|\mathbf{X}_{\text {part 1 }}-\mathbf{X}_{\text {part 2 }}\right\|\right)
$$

where $\sigma$ is the surface tension, $L_{\text {cap }}=\sqrt{\frac{\sigma}{\rho_{\text {fluid }} g}}(\mathrm{~m})$ is the capillary length. $\rho_{\text {fluid }}$ is the density of water and $g$ is the gravitational constant. For a temperature of $25^{\circ} \mathrm{C}$ the capillary length of water is $L_{c a p}=2.7 \mathrm{~mm} . K_{1}$ is the modified Bessel function of the second kind, first order. The operator $\|\mathbf{X}\|$ refers to the norm of vector $\mathbf{X}$. One of the assumptions here to ensure the validity of the model is that particles should not be very close to each other (typically not closer than the sum of the particles' radii). 


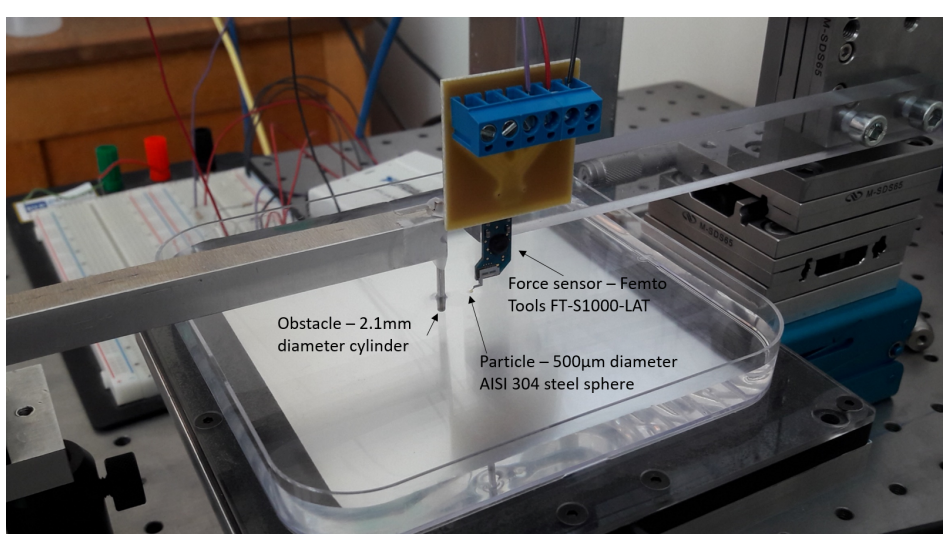

Fig. 3 Force measurement setup: a Femto-Tools force sensor measures the force between a $500 \mu \mathrm{m}$ diameter spherical steel particle and a $2.1 \mathrm{~mm}$ diameter cylindrical obstacle (the particle is glued onto the sensor)

Equation (1) was experimentally validated using the experimental setup shown in Fig. 3. A spherical AISI 304 steel sphere with $500 \mu \mathrm{m}$ diameter was glued on a Femto-Tools FT-S1000-LAT $(50 \mathrm{nN}-1000 \mu \mathrm{N})$ force sensor, and approached from a IPL-780 resin $2.1 \mathrm{~mm}$ diameter perturbing cylinder. Since the perturbation arises from the free surface deformation by the perturbing particle (characterized by the capillary charge), equivalence can be achieved by considering equal capillary charge $Q_{i}$ for both situations. The cylinder diameter was selected such that the resulting lateral capillary force $F_{\text {LatCap }}$ could be in the range of the force sensor.

Fig. 4 compares Equation (1) with the experimental lateral capillary force $F_{\text {LatCap }}$ as a function of the obstacle-particle distance $r_{\text {obst-part }}$. The theoretical model provides a good estimate for obstacle-particle distances larger than $3 \mathrm{~mm}$, and is inaccurate for smaller distances. However, as stated before, the model is valid for particle-obstacle distances larger than the sum of the radius of the particle $(250 \mu \mathrm{m})$ and of the radius of the cylinder $(1 \mathrm{~mm})$. The model is thus still valid when the distance between the two particles is greater than around $1.5 \mathrm{~mm}$. The inaccuracy of the model when the distance between the particles is between $1.5 \mathrm{~mm}$ and $3 \mathrm{~mm}$ comes from the fact that it is difficult to estimate the value of the capillary dipole $Q_{1}$ and $Q_{2}$. To avoid computing these parameters, a fitted model is proposed. The experimental data presented in Fig. 4 can be used to estimate the parameters in Eq. 1 which gives the expression of the lateral capillary force $F_{\text {LatCap }}$. A least square fit of the experimental measurement is performed to obtain the following empirical fitted model which is then used to design the feedforward controller (Section 4):

$$
F_{\text {LatCap }}=9.9 \cdot 10^{-6} K_{1}\left(800.56\left\|\mathbf{X}_{\text {obst-part }}\right\|\right)
$$

where $\mathbf{X}_{\text {obst-part }}$ is the center-to-center obstacle-particle vector $[\mathrm{m}]$ and the resulting force $F_{\text {LatCap }}$ is given in N. 


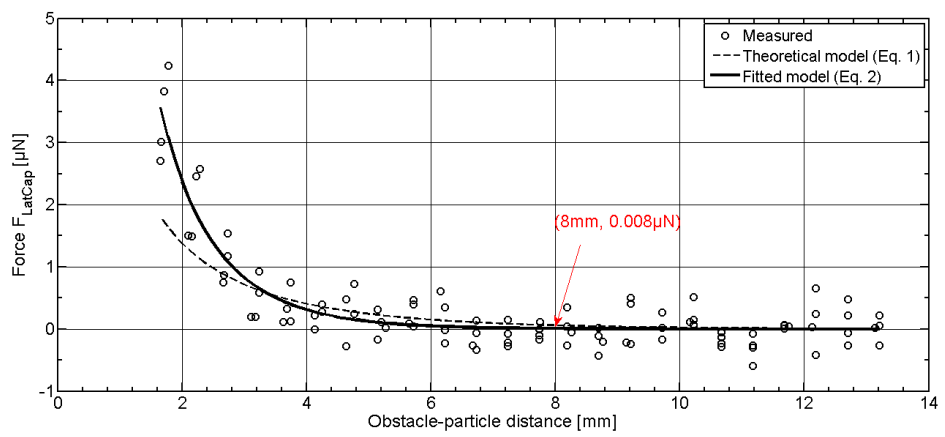

Fig. 4 Lateral capillary forces between the spherical particle and the cylindrical obstacle. Circles indicate Femto-Tools measurements. Dashed line the theoretical model of 1 without any fit: capillary charges are estimated from contact angles and wetting radius. Solid line is the fitted model reported in 2 , introduced to better estimate the force at small obstacleparticle distance. Typically, as discussed later on in Section 5.3, the force is estimated at about $8 \mathrm{nN}$ for a particle-obstacle distance of $8 \mathrm{~mm}$ (Adapted from [23])

When the particle is displaced towards the obstacle, it undergoes on one hand the viscous drag of the fluid and on the other hand the lateral capillary force. At a certain distance from the obstacle the velocity of the particle is null as the lateral capillary force, which repels the particle, is balanced by the drag force induced by the fluid velocity. The particle cannot be manipulated closer than this equilibrium point since the thermocapillary force is not strong enough to overcome the lateral capillary force. The thermocapillary actuation force generated by the laser was shown to be about $10 \mathrm{nN}$ [12]. This result is obtained by simulating and measuring the flow velocity, and converting this data to a force using the Stokes' law which gives an approximation of the drag force applied to a particle staying at the interface. From Fig 4, this corresponds to a distance of $10 \mathrm{~mm}$. In the following the distance between the particle and the cylinder will thus be kept greater than $10 \mathrm{~mm}$.

In the context of the manipulation of several particles it can also be interesting to know the minimal distance that should be maintained between two $500 \mu \mathrm{m}$ diameter spheres. As previously the thermocapillary actuation force generated by the laser is $10 \mathrm{nN}$. According to the model (Eq. 1), the lateral capillary force between two $500 \mu \mathrm{m}$ diameter steel spheres is $F_{\text {LatCap }} \approx 9.95 \mathrm{nN}$ at a distance of $4.7 \mathrm{~mm}$. The distance between these two particles should thus be larger than $4.7 \mathrm{~mm}$. This limitation could be removed by increasing the laser power.

\section{Control of the Thermocapillary System}

The goal of the controller is to move the particle to a given target $X_{\text {targ }}$. The thermocapillary flows actually push particles, which makes the system unstable. Due to small perturbations, the particle velocity is likely to deviate 
from the current target direction (Fig. 5). A closed loop controller has to be implemented to guarantee the robustness of the system to perturbations, such as vibrations or air flows. It is also designed to avoid obstacles placed at the air/liquid interface.

The proposed controller is composed of a feedback loop, aiming at increasing the robustness of the control with respect to perturbations, and a feedfoward loop to avoid obstacles (Fig. 6).

The output of the controller is the particle position $X_{\text {part }}$. It is obtained from the images taken by the camera. Based on the measured particle position $\mathbf{X}_{\text {part }}$ and particle velocity $\dot{\mathbf{X}}_{\text {part }}$, the closed loop controller shown in Fig. 6 computes the velocity correction $\dot{\mathbf{X}}_{\text {part }}^{\mathrm{c}}$ to realign the particle velocity towards the target. The particle is conveyed by the fluid flow, onset by heating the fluid with the laser. Thus, the controller determines the desired position of the laser $\mathbf{X}_{\text {lasD }}$ to reach the target location. A mirror is used to modify the position of the laser spot on the surface of the water. It is actuated by two voltages. These two voltages are the input of the system.

The detailed controller is presented in Fig. 9. As shown in [13], the transfer function of the Controlled Mirror system is considered to be equal to identity so only the control of the thermocapillary system has to be recalled here.

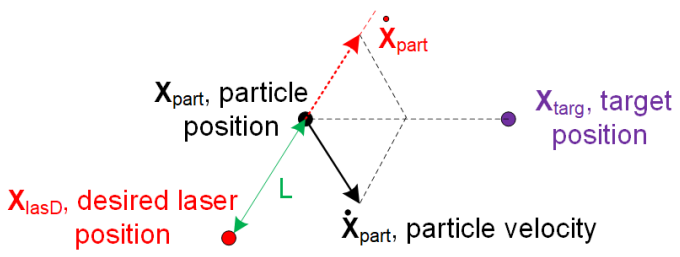

Fig. 5 The particle position $X_{\text {part }}$ is given by image analysis, hence its velocity $\dot{X}_{\text {part }}$ can be calculated from two successive snapshots. The desired correcting action of the laser is then evaluated to correct any deviation from the target direction, and the proposed algorithm locates the next laser position with respect to the particle position (distance $L$ and direction).

As shown later on in Fig. 9, the particle velocity magnitude and direction are actually decoupled, leading to the steady-state particle velocity magnitude $\hat{\dot{r}}_{\text {part QSS }}$ and direction $\theta_{\text {corr }}$. The targeted laser position $\mathbf{X}_{\text {lasD }}$ is obtained from the inverse model of the empirical characteristics [13] $\left(r_{\text {las-part }}\right.$ in mm, $\hat{\dot{r}}_{\text {part QSS }}$ in $\left.\mathrm{mm} \mathrm{s}^{-1}\right)$ :

$$
r_{\text {las-part }}=-0.732 \hat{\dot{r}}_{\text {part QSS }}+4.177
$$

The orientation $\theta_{\text {las-part }}$ of the laser-particle vector $\mathbf{X}_{\text {las-part }}$ is then computed based on the correction angle $\theta_{\text {corr }}$

$$
\theta_{\text {las-part }}=\theta_{\text {part-targ }}-\theta_{\text {corr }}
$$

where $\theta_{\text {part-targ }}$ is the desired orientation, the orientation of the particle-target vector $\mathbf{X}_{\text {part-targ }}$. 


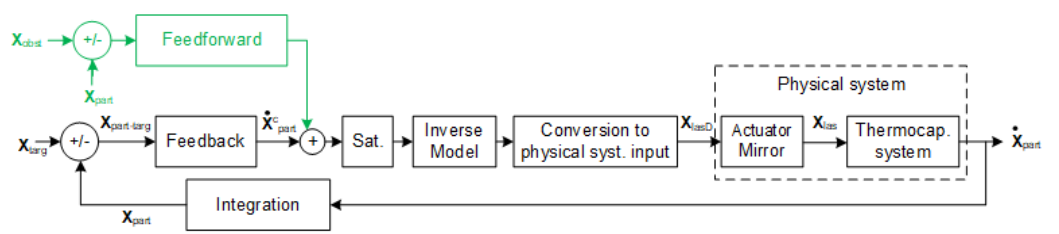

Fig. 6 Simplified schematic representation of the control scheme (Reproduced with permission from [23])

The laser-particle relative position $\mathbf{X}_{\text {las-part }}$ is computed from the laserparticle distance $r_{\text {las-part }}$ and laser-particle orientation $\theta_{\text {las-part }}$ (this is done in the "Vector Comp" block shown in Fig. 9). As a result, the corresponding desired laser position $\mathbf{X}_{\text {lasD }}=\mathbf{X}_{\text {part }}-\mathbf{X}_{\text {las-part }}$ is then computed.

This controller ensures the robustness with respect to perturbations. However, in case obstacles should be avoided, a feedforward controller (green box in Fig. 6 and Fig. 9) should be added to the system. It aims at rejecting the disturbance due to the lateral capillary force. This feedforward controller is discussed in the following. As sketched in Fig. 7, the disturbing capillary

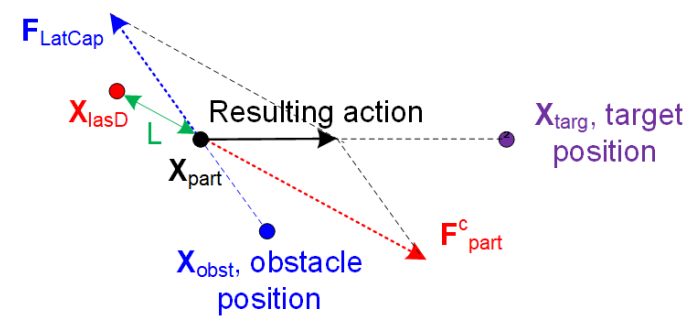

Fig. 7 Based on the measured particle and obstacle positions, a disturbing force $F_{\text {LatCap }}$ is computed from (2) and converted into a disturbing velocity from (5), which can be compensated with an appropriate laser position to realign the particle velocity towards the target.

force is calculated with (2) from the obstacle-particle distance. This disturbing force $F_{\text {LatCap }}$ is then converted into a disturbing velocity using the Stokes' Law (commonly used to compute a viscous force from a particle velocity, according to [27], [28], [29]). In other words, an equivalent velocity $\hat{\dot{r}}_{\text {part }}$ QSS FF is computed, which is proportional to the disturbing force:

$$
\hat{\dot{r}}_{\text {part QSS FF }}=\frac{F_{\text {LatCap }}}{6 \pi \mu R_{\text {part }}}
$$

where $\mu$ is the liquid dynamic viscosity and $R_{\text {part }}$ the particle radius. While using the Stokes' Law, [30] showed that the classical expression must be corrected by a drag coefficient (called here after a prefactor) to account for the presence of the particle at the surface and not in the bulk of the liquid. This 
prefactor depends on the particle wetting properties, so a linear relationship is preserved between velocity and force. Since we are interested in performing closed loop control, the exact value of the prefactor is not important, because it can be compensated by adapting the proportional correction of the controller. This prefactor does not change the order of magnitude of the viscous drag, as can be concluded from the comparison between theoretical (based on this drag model) and experimental force discussed in this paper.

Beside the disturbance magnitude, a correction angle $\theta_{\text {corr FF }}$ is also computed in the "FF Direction action" block of Fig. 9 using the following relation:

$$
\theta_{\text {corr FF }}=\theta_{\text {part-targ }}-\theta_{\text {obst-part }}
$$

This equation represents the direction opposite on which the lateral capillary force is acting with respect to the particle-target direction $\theta_{\text {part-targ }}$.

Hence, the feedforward controller estimates the particle velocity at quasi steady-state $\hat{\dot{r}}_{\text {part }}$ QSS FF and calculates a correction angle $\theta_{\text {corr FF }}$ (FF denotes the values of the inputs computed by the feedforward controller) based on the measured obstacle-particle position $\mathbf{X}_{\text {obst-part }}$. The system inputs computed by the feedforward and feebdack controllers are then added, as shown in Fig. 6 . Using these resulting values, a desired laser position $\mathbf{X}_{\text {lasD }}$ is computed using the linearization procedure recalled in the previous subsection.

The feedforward controller was designed with an inversion based approach, which enabled to define $\hat{\dot{r}}_{\text {part }}$ QSS FF computed in the "FF Mag action" block (green box in Fig. 6). This block is fed with the obstacle-particle distance $r_{\text {obst-part }}$ which is used to compute the Cheerios force $F_{\text {LatCap }}$ acting on the particle using eq. 2. The inputs computed by both controllers are added in the "Input combinator" block as if they were defining a vector with polar coordinates: $\hat{\dot{r}}_{\text {part }}$ QSS xx and $\theta_{\text {corr xx }}$, according to equations (7-8) on page 12 .

The resulting $\hat{\dot{r}}_{\text {part }}$ QSS (once limited to $4.5 \mathrm{~mm} / \mathrm{s}$ in the saturation block) and $\theta_{\text {corr }}$ are then used to compute a desired laser position $\mathbf{X}_{\text {lasD }}$. The latter one allows reaching the goals of the feedback and feedforward controllers: the controller is robust to perturbations, and enables avoiding obstacles. Indeed, for strong capillary forces (ie particles close to the obstacle), the feedforward controller dominates the feedback controller. Conversely, for particles far away from the obstacle, the laser position $\mathbf{X}_{\text {lasD }}$ leads to a total radial force displacing the particle towards the target location $\mathbf{X}_{\mathrm{targ}}$.

\section{Results}

\subsection{Simulation Results}

The control strategy was first tested in simulation using the Matlab/Simulink software. The starting and target positions of the sphere are respectively $(0$, $-20) \mathrm{mm}$ and $(0,20) \mathrm{mm}$ (fig. 8). The disturbing cylinder is located in $(7,0)$ $\mathrm{mm}$. 


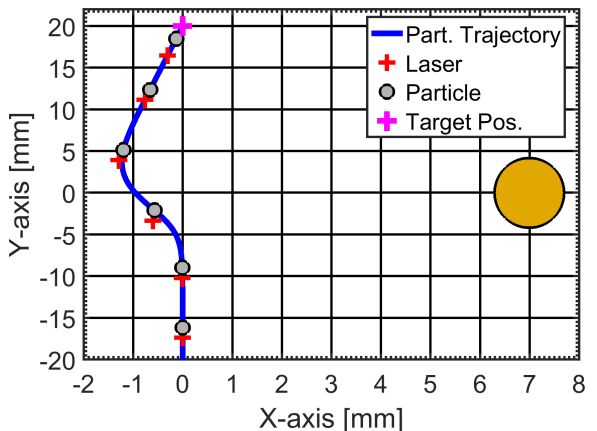

(a) Feedforward controller turned off

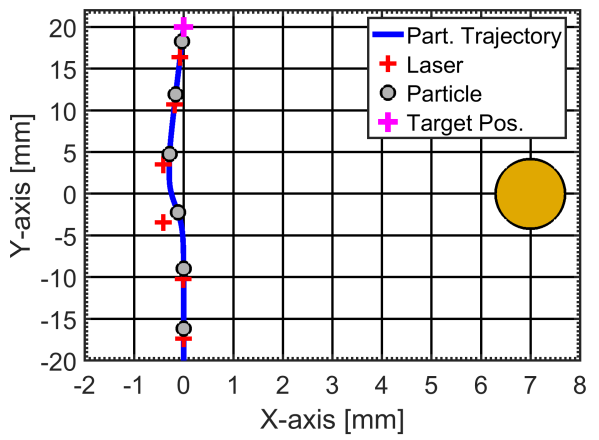

(b) Feedforward controller turned on

Fig. 8 Simulation results of a controlled manipulation of a $500 \mu \mathrm{m}$ diameter steel sphere in presence of a cylindrical obstacle. To show the effect of the controllers, the laser and particle positions are plotted for the time instants $t=1,2.6,4.2,5.8,7.4,9.0 \mathrm{~s}$ (Adapted from [23]). 


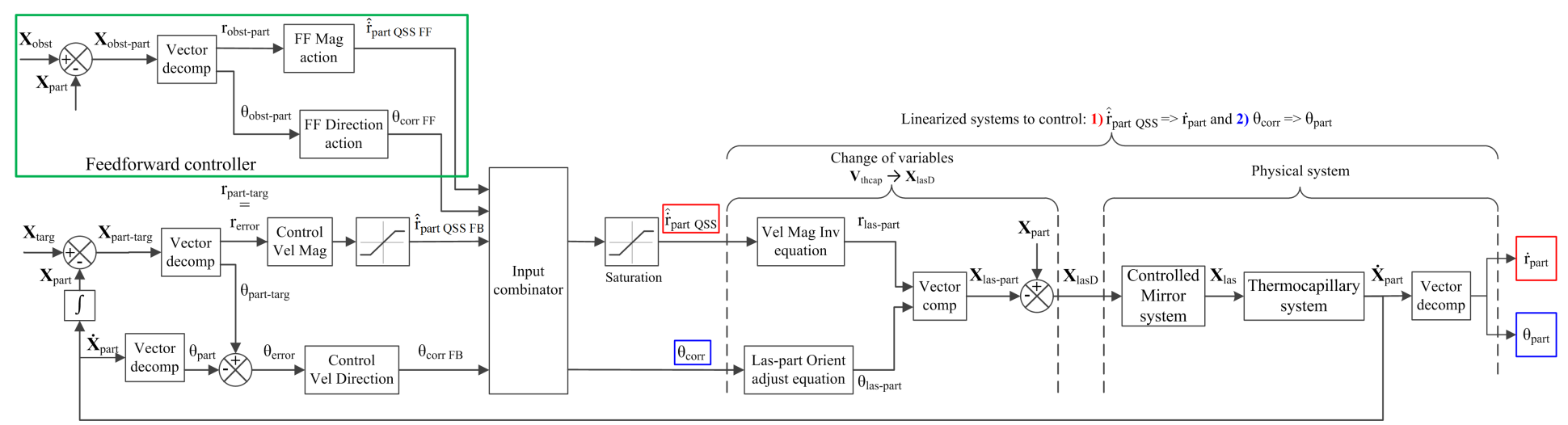

Fig. 9 Block diagram representation of the entire closed-loop system with the feedforward controller. This controller has two outputs: an estimated particle velocity magnitude at quasi steady-state $\hat{\dot{r}}_{\mathrm{part}} \mathrm{OSS} \mathrm{FF}$ and a correction angle $\theta_{\text {corr }} \mathrm{FF}$. These outputs are combined with the outputs of the

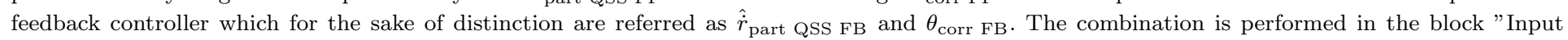
combinator" which computes the estimated particle velocity magnitude at quasi steady-state $\hat{\dot{r}}_{\text {part }}$ QSS and the correction angle $\theta_{\text {corr }}$ FB which are used to compute the desired laser position $\mathbf{X}_{\text {lasD }}$ (Reproduced with permission from [23]).

$$
\begin{aligned}
\hat{\dot{r}}_{\text {part QSS }} & =\sqrt{\hat{\dot{r}}_{\text {part QSS FB }}^{2}+\hat{\dot{r}}_{\text {part QSS FF }}^{2}+2 \hat{\dot{r}}_{\text {part QSS FB }} \hat{\dot{r}}_{\text {part QSS FF }} \cos \left(\theta_{\text {corr FF }}-\theta_{\text {corr FB }}\right)} \\
\theta_{\text {corr }} & =\theta_{\text {corr FB }}+\arctan \left(\frac{\hat{\dot{r}}_{\text {part QSS FF }} \sin \left(\theta_{\text {corr FF }}-\theta_{\text {corr FB }}\right)}{\hat{\dot{r}}_{\text {part QSS FB }}+\hat{\dot{r}}_{\text {part QSS FF }} \cos \left(\theta_{\text {corr FF }}-\theta_{\text {corr FB }}\right)}\right)
\end{aligned}
$$


The model for the particle velocity magnitude $\dot{r}_{\text {part }}$ can be seen as a cascade of a non-linear steady-state characteristics and a linearly time-variant dynamics. The first one is given by :

$$
\hat{\dot{r}}_{\text {part QSS }}=26.086 \exp \left(-1.433 r_{\text {las-part }}\right)
$$

The second one links the steady-state output $\hat{\dot{r}}_{\text {part }}$ QSS to the particle velocity $\dot{r}_{\text {part }}$, according to the following second-order transfer function:

$$
\operatorname{VelDyn}(s) \equiv \frac{\dot{r}_{\text {part }}}{\hat{\dot{r}}_{\text {part QSS }}}=\frac{52.027}{s^{2}+7.6458 s+52.0274}
$$

The reason why the model relating the particle velocity to the distance laser particle includes this second order transfer function is to take into account the inertia of the fluid, which induces a transient time on the particle velocity.

The feedforward controller was first turned off. We can see in Fig. 8(a) that the laser primarily aims at pushing the particle towards the target location. Consequently, the $\mathrm{X}$-axis deviation can reach up to $1.22 \mathrm{~mm}$ in the worst case. The feedforward controller was then turned on to counteract the effect of the lateral capillary force $F_{\text {LatCap }}$ while also displacing the particle towards the target location. As a result (Fig. 8(b)), the particle is only deviated by a distance of $29 \mu \mathrm{m}$ in the $\mathrm{X}$-axis. The lateral capillary force $F_{\text {LatCap }}$ could however not be counteracted completely, because of saturation of the actuator (the perturbation has been voluntarily chosen large enough to be measured, and therefore a bit too large for the laser power. The control loop therefore introduced a saturation, ie a minimal distance from the controlled particle to prevent the laser to spot i.e. too close from it).

\subsection{Experiments}

Experimental results were obtained with a AISI 304 steel $500 \mu \mathrm{m}$ diameter particle floating on ultra pure water in a plastic container placed above a white LED panel improving the particle position acquisition in real time with a camera. The laser is moved by a mirror using piezoelectric actuators [13]. As already devised, the perturbation is produced with a fixed IPL-780 resin cylinder (2.1 mm diameter, produced by Nanoscribe Photonics GT). The start and target points have the same $Y$ coordinate approximately only because it is not possible to control exactly the initial position of the floating particle. On its trajectory to the target, the closest point from the cylinder is at $12.8 \mathrm{~mm}$. As shown in Fig. 10, a maximum deviation of $3.05 \mathrm{~mm}$ is observed without the feedforward while it has been reduced to $1.27 \mathrm{~mm}$ with the feedforward activated. As a final note, let us mention that the controller is turned off when the particle enters a $250 \mu \mathrm{m}$ circle centered on the targeted position. 


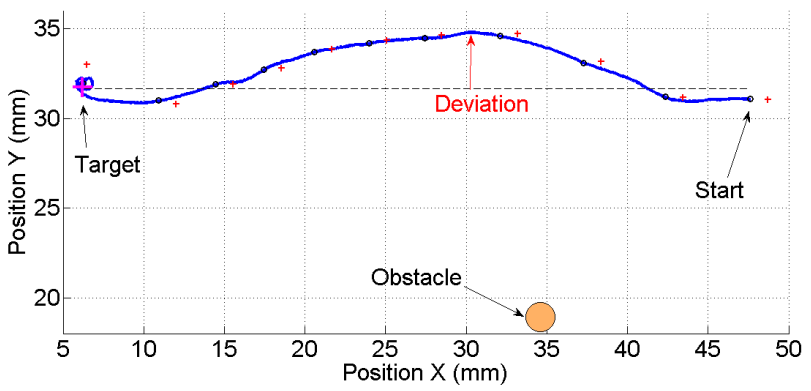

(a) Feedforward controller turned off

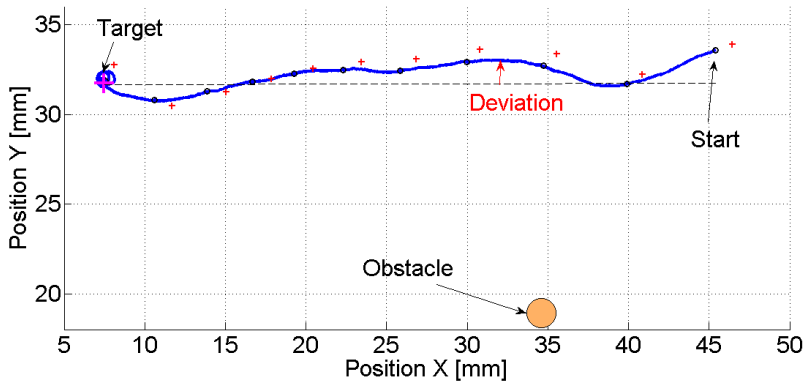

(b) Feedforward controller turned on

Fig. 10 Step response of the particle in the presence of an obstacle. The particle starts at the right of the cylinder and is controlled towards a target position (magenta cross) with a $\mathrm{Y}$ coordinate similar to its origin. The laser (red cross) and particle (black circles) positions are plotted for the time instants $t=0,3,6,9,12,15,18,21,24,27,30 \mathrm{~s}$ (Adapted from [23]).

\subsection{Thermocapillary Force Estimation}

A theoretical estimation of the thermocapillary force developed by the laser and applied by the liquid on the particle has been reported in [13]. First, the liquid flow velocity was simulated with Comsol (note that an experimental validation of the order of magnitude has been done with PIV and is reported in [12]). Second, this flow velocity was converted into a force with a viscous Khan and Richardson's law (similar to Stokes' law). The force was estimated to be about $10 \mathrm{nN}$.

An experimental estimation of this $10 \mathrm{nN}$ order of magnitude is obtained as follows. The thermocapillary micromanipulation setup described here above can be used to push the $500 \mu \mathrm{m}$ diameter particle towards the $2 \mathrm{~mm}$ diameter cylindrical obstacle. The laser power is set at $37 \mathrm{~mW}$, and the laser-particle distance set at $1.1 \mathrm{~mm}$. The particle was pushed 3 times from different starting points, and this was repeated 3 times with 3 different samples of water (the water was removed from the tank and fresh ultra pure water was poured at each attempt). The trajectories were recorded and are displayed in Figure 11. 


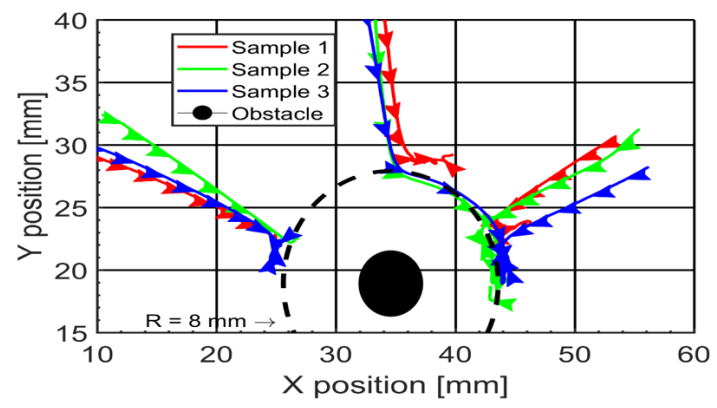

Fig. 11 During this experiment, the $500 \mu \mathrm{m}$ diameter steel sphere is pushed by the thermocapillary flow towards the black circle which represents the $2 \mathrm{~mm}$ diameter cylindrical obstacle. Each color indicates the three trials made from three different starting points of the particle, and each color represents a repetition with a different water sample.

It can be seen on this Figure that the laser is not strong enough to push the particle inside a $8 \mathrm{~mm}$ shielding circle centered on the obstacle. Reporting this $8 \mathrm{~mm}$ particle-obstacle distance in Figure 4 (i.e. in equation (2)), the Cheerios force is estimated to be about $8 \mathrm{nN}$, which confirms the order of magnitude of the thermocapillary force, since the thermocapillary force and Cheerios force balance one another for this particle-obstacle distance.

\section{Discussion and Conclusions}

This paper recalled results recently published in [23], based on a feedforward control scheme able to compensate the disturbance Cheerios force exerted on a particle by a disturbing neighboring particle or obstacle. This is indeed a first step towards handling multiple particles simultaneously, avoiding floating particles from attracting one another. In terms of handling strategies, future work will focus on multiple handling, but also on the angular control of non radially symmetric objects. The automatic handling tool was also used to estimate experimentally the level of the thermocapillary force developed by the laser. This is the beginning in better understanding and maybe modelling the physical system. Up to now, the physical system has to be identified experimentally for each modification of the laser, liquid or particle parameters (this identified model is indeed used in the control scheme). A refined experimental characterization of the thermocapillary force could simplify this tedious characterization in the future.

Acknowledgements Supported by 7/38 MicroMAST IAP (Belspo, Belgium) and by the EIPHI Graduate School (contract "ANR-17-EURE-0002"). 


\section{References}

1. A. Oulmas, N. Andreff, S. Régnier, 2017 IEEE/RSJ International Conference on Intelligent Robots and Systems (IROS) pp. 1877-1882 (2017)

2. V. Gauthier, A. Bolopion, M. Gauthier, Micromachines 8(8) (2017)

3. Q. Zhou, V. Sariola, K. Latifi, V. Liimatainen, Nature Communications 7, 12764 (2016). DOI 10.1038/ncomms12764

4. M. Yin, E. Gerena, C. Pacoret, D.S. Haliyo, S. Régnier, 2017 IEEE/RSJ International Conference on Intelligent Robots and Systems (IROS) pp. 1889-1894 (2017)

5. Terrazas Mallea, Ronald and Bolopion, Aude and Beugnot, Jean-Charles and Lambert, Pierre and Gauthier, Michael, in Intelligent Robots and Systems (IROS) (2017), pp. 408-413

6. E. Vela, Non-contact microscale manipulation using laser-induced convection flows. Ph.D. thesis, Paris 6 (2010)

7. Winterer, Felix and Maier, Christoph $\mathrm{M}$ and Pernpeintner, Carla and Lohmüller, Theobald, Soft matter 14(4), 628 (2018)

8. E. Vela, C. Pacoret, S. Bouchigny, S. Regnier, K. Rink, A. Bergander, in Intelligent Robots and Systems (IROS) (2008), pp. 913-918. DOI 10.1109/IROS.2008.4650918

9. E. Munoz, J. Quispe, P. Lambert, A. Bolopion, R. Terrazas, S. Régnier, E. Vela, J Micro-Bio Robot 12, 65 (2017)

10. W. Hu, Q. Fan, A.T. Ohta, Robotics and Biomimetics 1(1), 1 (2014). DOI 10.1186/s40638-014-0014-3

11. W. Hu, Q. Fan, A.T. Ohta, Lab Chip 13(12), 2285 (2013). DOI 10.1039/C3LC50389E

12. R.T. Mallea, Thermocapillary micromanipulation: laser induced convective flows towards controlled handling of particles at the free surface. Ph.D. thesis, Université libre de Bruxelles / Université de Franche-Comté (2017)

13. Terrazas Mallea, Ronald and Bolopion, Aude and Beugnot, Jean-Charles and Gauthier, Michael and Lambert, Pierre, IEEE/ASME Transactions on Mechatronics 22(2), 693 (2017)

14. Ng, Jessamine M. K. and Fuerstman, Michael J. and Grzybowski, Bartosz A. and Stone, Howard A. and Whitesides, George M., Journal of the American Chemical Society 125(26), 7948 (2003). DOI 10.1021/ja0347235. URL http://dx.doi.org/10.1021/ja0347235. PMID: 12823016

15. Alexander Jesacher and Severin Fürhapter and Christian Maurer and Stefan Bernet and Monika Ritsch-Marte, Opt. Express 14(13), 6342 (2006). DOI 10.1364/OE.14.006342. URL http://www.opticsexpress.org/abstract.cfm?URI=oe-14-13-6342

16. Shweta Gupta and Nahar Singh and Murali Sastry and Rita Kakkar and Renu Pasricha, Thin Solid Films 519(3), 1072 (2010). DOI http://dx.doi.org/10.1016/j.tsf.2010.08.046. URL http://www.sciencedirect.com/science/article/pii/S0040609010011612. Biomolecular Electronics and Organic Nanotechnology for Environmental Preservation

17. Rogers, Priscilla and Gralinski, Ian and Galtry, Cameron and Neild, Adrian, Microfluidics and Nanofluidics 14(3-4), 469 (2013). DOI 10.1007/s10404-012-1065-9. URL http://dx.doi.org/10.1007/s10404-012-1065-9

18. Dkhil, M. and Kharboutly, M. and Bolopion, A. and Regnier, S. and Gauthier, M., IEEE Transactions on Automation Science and Engineering 14(3), 1387 (2017). DOI 10.1109/TASE.2015.2448133

19. Terrazas Mallea, Ronald and Bolopion, Aude and Beugnot, Jean-Charles and Gauthier, Michael and Lambert, Pierre, IEEE/ASME Transactions on Mechatronics 23(4), 8371646 (2018)

20. Rahman, M Arifur and Takahashi, Noboru and Siliga, Kawai F and Ng, Nigel K and Wang, Zhidong and Ohta, Aaron T, Robotics and Biomimetics 4(1), 7 (2017)

21. Rahman, Mohammad Arifur and Wang, Zhidong and Ohta, Aaron T, Micro \& Nano Letters 12(11), 891 (2017)

22. Rahman, M Arifur and Cheng, Julian and Wang, Zhidong and Ohta, Aaron T, Scientific Reports 7(1), 3278 (2017)

23. R. Terrazas Mallea, A. De Maeijer, A. Bolopion, M. Gauthier, M. Kinnaert, P. Lambert, in MARSS conference (2018) 
24. J. Xu, K.W. Plaxco, S.J. Allen, The Journal of Chemical Physics 124(3), 036101 (2006). DOI http://dx.doi.org/10.1063/1.2151267. URL http://scitation.aip.org/content/aip/journal/jcp/124/3/10.1063/1.2151267

25. Marmur, Abraham and Ras, Robin H. A., Soft Matter 7, 7382 (2011). DOI 10.1039/C1SM05156C. URL http://dx.doi.org/10.1039/C1SM05156C

26. P.A. Kralchevsky, K. Nagayama, Chapter 7 - Lateral Capillary Forces between Partially Immersed Bodies (Elsevier, 2001), Studies in Interface Science, vol. 10, pp. 287 - 350. DOI https://doi.org/10.1016/S1383-7303(01)80048-X

27. R.A. Flynn, A.L. Birkbeck, M. Gross, M. Ozkan, B. Shao, M.M. Wang, S.C. Esener, Sensors and Actuators B: Chemical 87(2), 239 (2002)

28. S. Chowdhury, P. Švec, A. Thakur, C. Wang, W. Losert, S.K. Gupta, in ASME 2013 International Design Engineering Technical Conferences and Computers and Information in Engineering Conference (2013), p. V001T09A031

29. M.C. Zhong, X.B. Wei, J.H. Zhou, Z.Q. Wang, Y.M. Li, Nature Communications 4, $1768(2013)$

30. Dorr, Aaron and Hardt, Steffen and Masoud, Hassan and Stone, Howard A., Journal of Fluid Mechanics 790, 607?618 (2016). DOI 10.1017/jfm.2016.41 